\title{
What Does Nitric Acid Really Do to Carbon Nanofibers?
}

S. Sainio ${ }^{1,}$, D. Nordlund ${ }^{2}$, R. Gandhiraman ${ }^{3}$, H. Jiang ${ }^{4}$, J. Koehne ${ }^{3}$, J. Koskinen ${ }^{5}$, M. Meyyappan ${ }^{3}$, and T. Laurila ${ }^{1, *}$

${ }^{1}$ Department of Electrical Engineering and Automation, School of Electrical Engineering, Aalto University, Espoo 02150, Finland

2 Stanford Synchrotron Radiation Lightsource, SLAC National Accelerator Laboratory, Menlo Park, CA 94025, United States

${ }^{3}$ Center for Nanotechnology, NASA Ames Research Center, Moffett Field, CA 94035, United States

${ }^{4}$ Department of Applied Physics, School of Science, Aalto University, Espoo 02150, Finland

${ }^{5}$ Department of Materials Science, School of Chemical Technology, Aalto University, Espoo 02150, Finland

\section{Supporting Information}




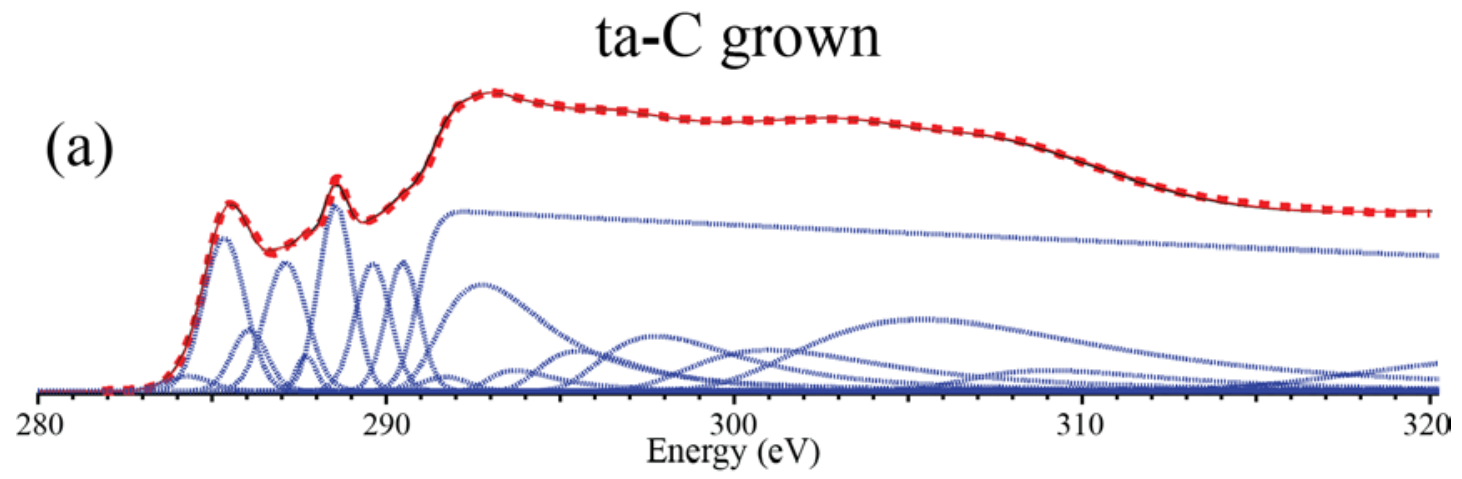

ta-C grown, $\mathrm{HNO}_{3}$ treated

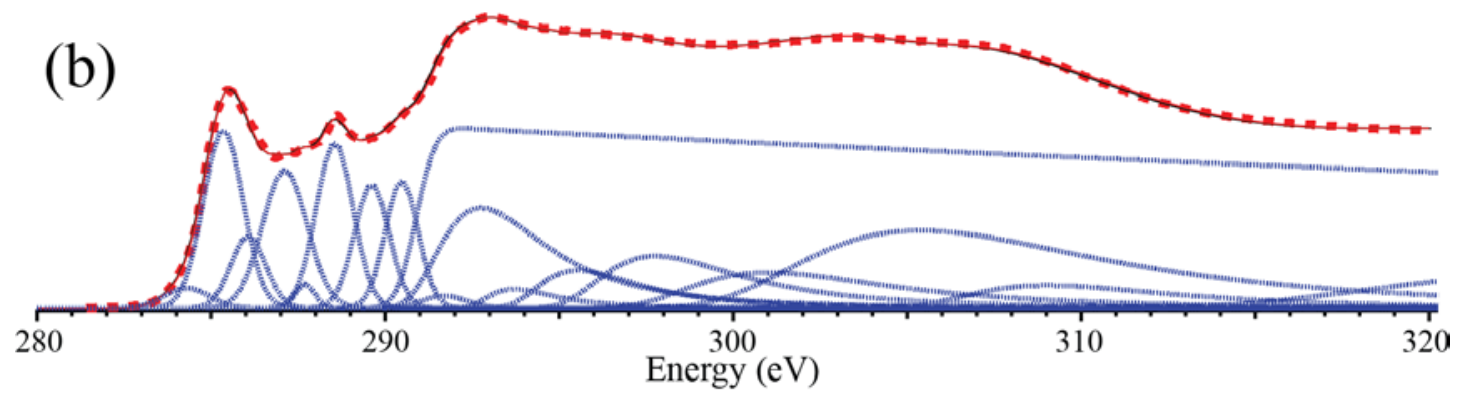

bamboo-like

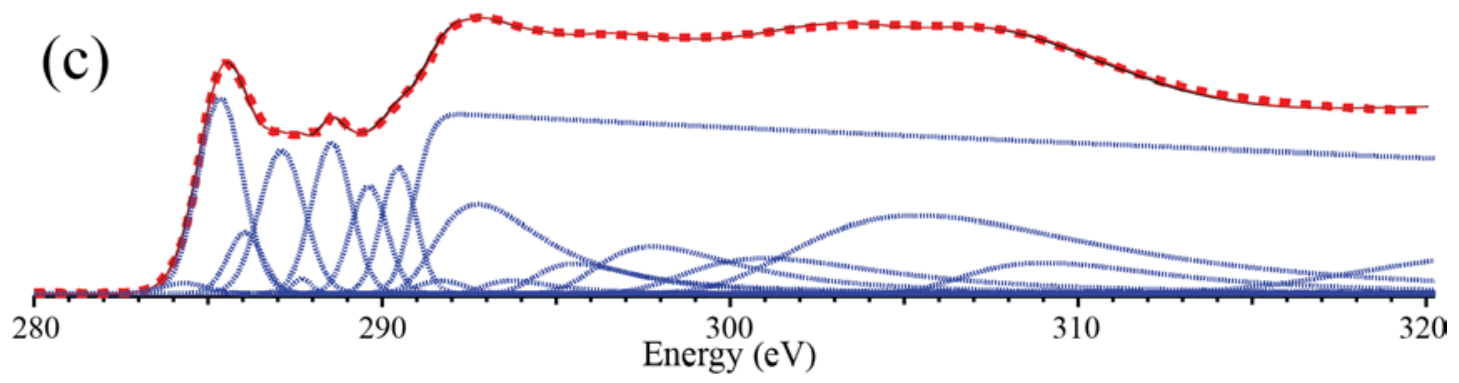

bamboo-like, $\mathrm{HNO}_{3}$ treated

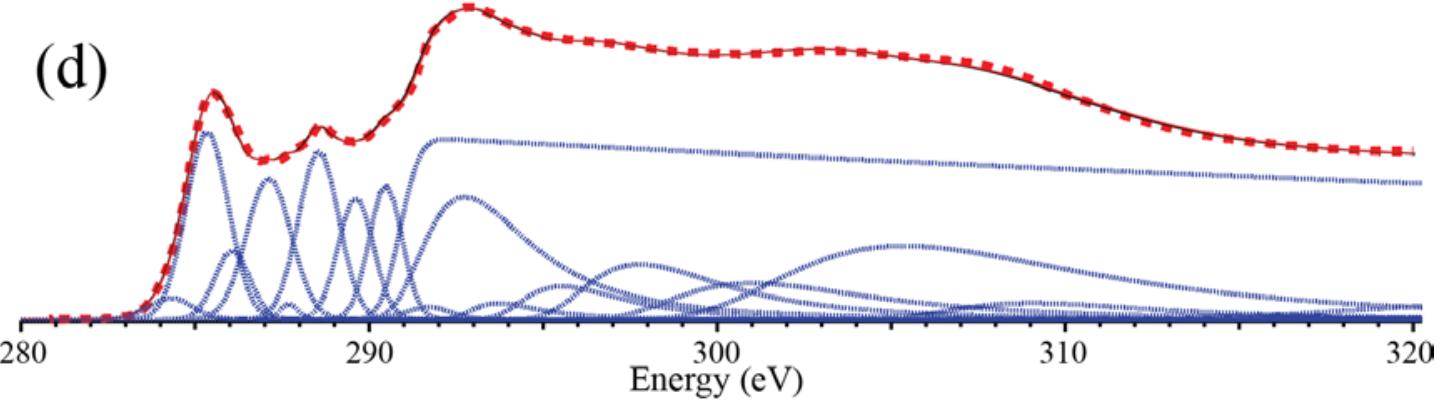

Figure S1: Fitted C1s spectra for both ta-C grown and bamboo-like fibers before and after the nitric acid treatment 


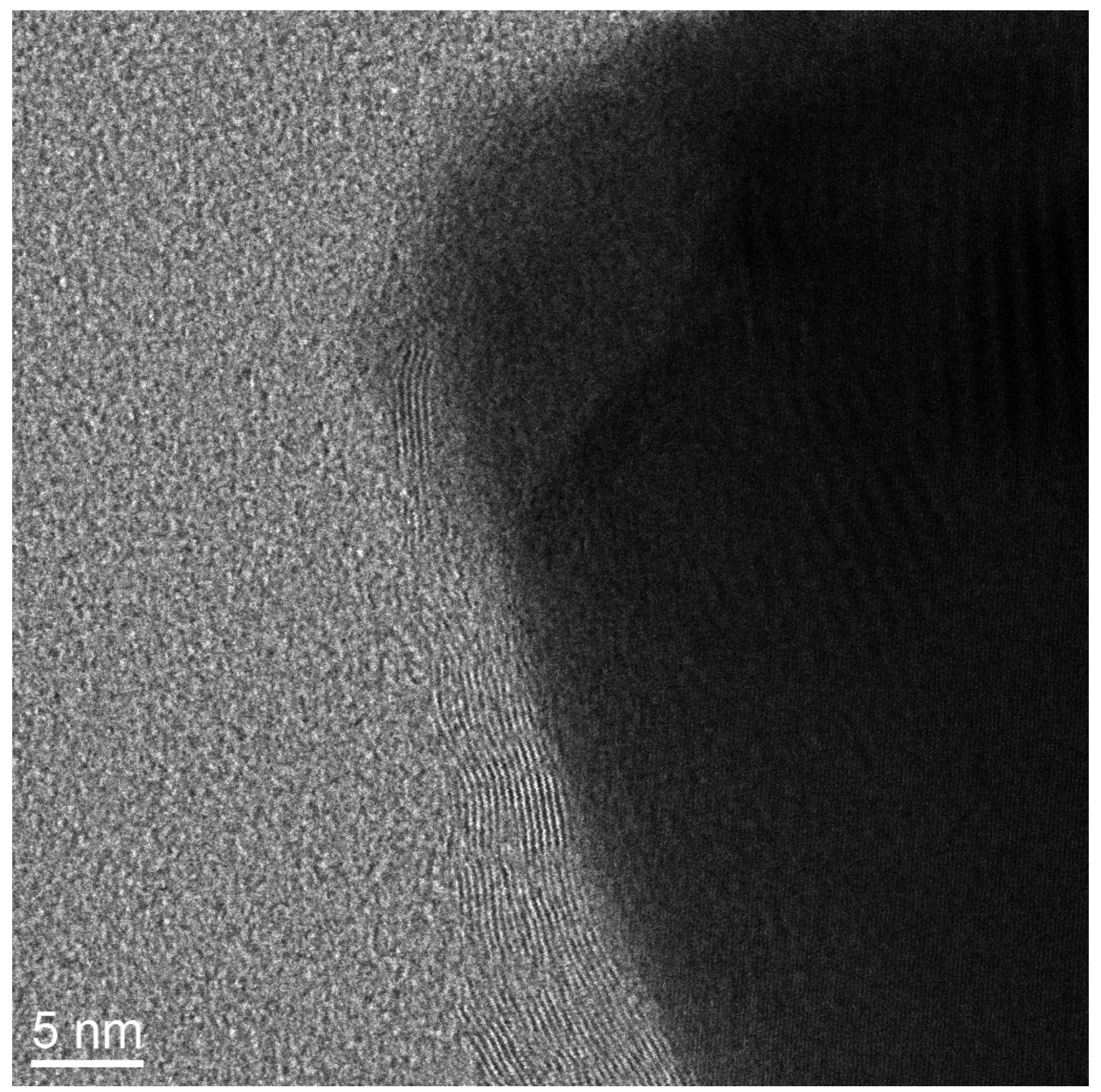

Figure S2: Graphite sheets aligned towards the Ni particle at the tip of the ta-C grown fiber after the nitric acid treatment 


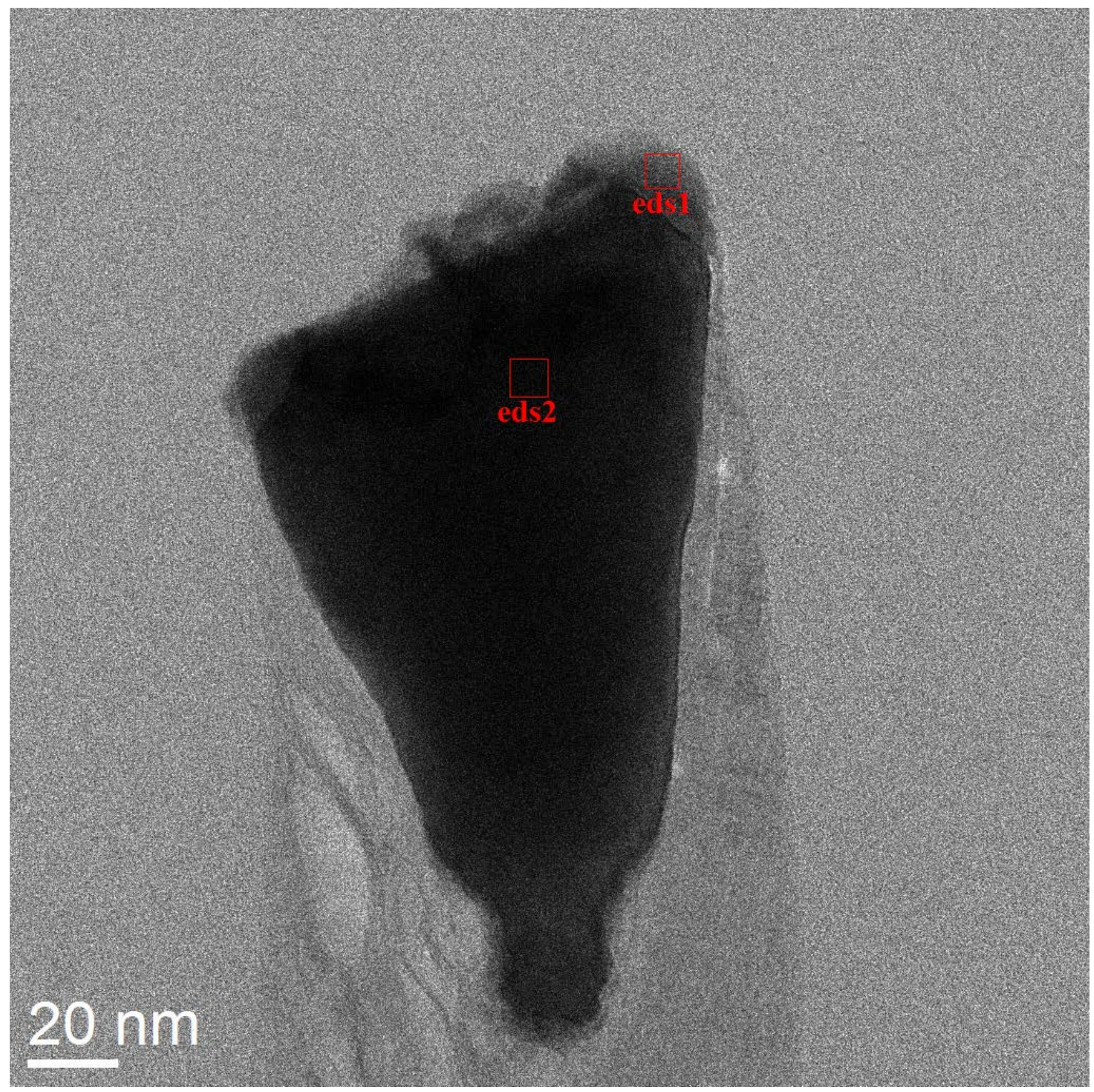

Figure S3: Ni particle at the tip of the ta-C grown fiber after the 5 minute $10 M$ nitric acid treatment. Locations for the EDS measurement areas are marked to the image 


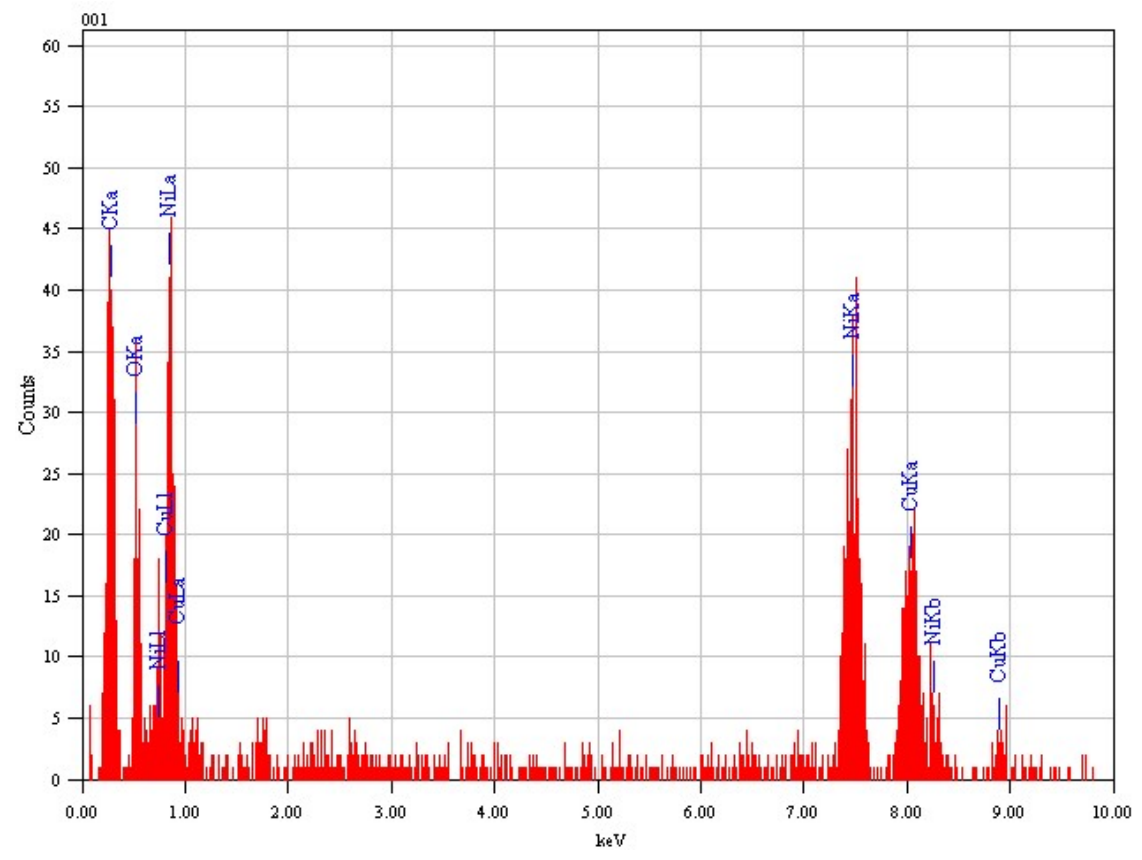

Figure S4: EDS spectra measured from the top edge of the Ni particle in the ta-C grown fiber (location marked in the Figure S3 as eds1)

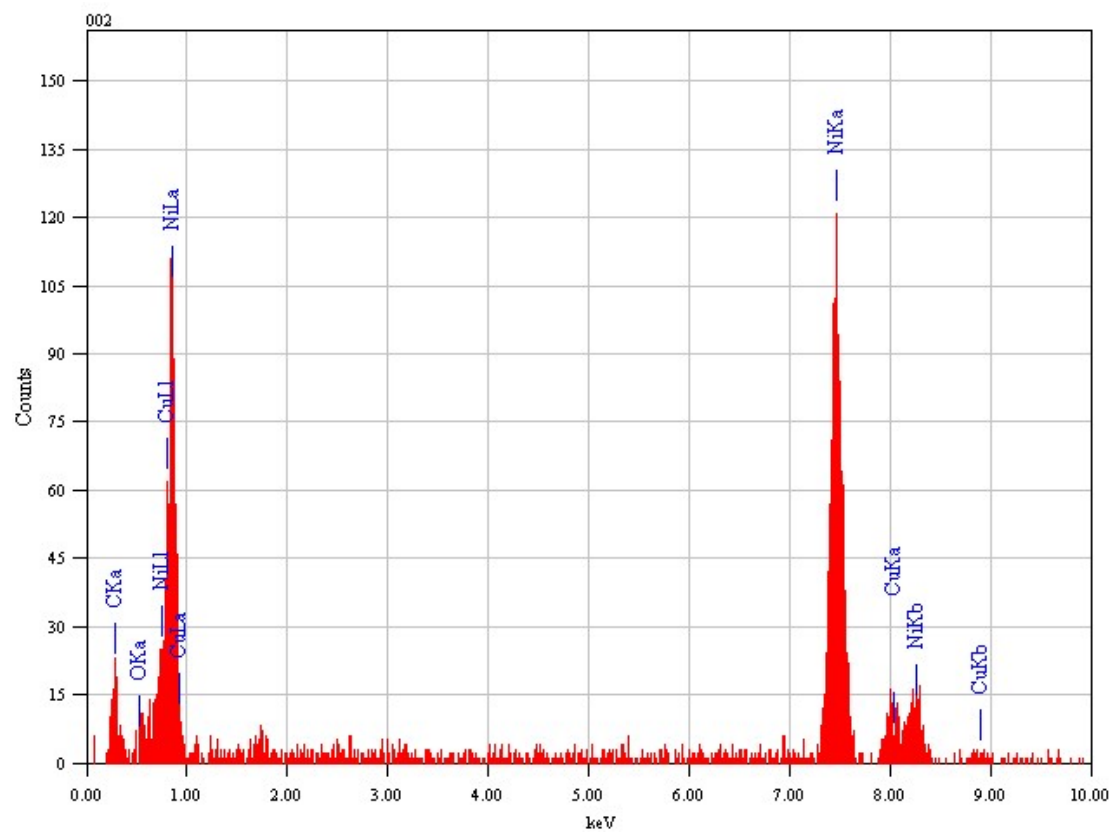

Figure S5: EDS spectra measured from the bulk of the Ni particle in the ta-C grown fiber (location marked in the Figure S3 as eds2) 